\title{
Management of Advanced Stage Hodgkin Lymphoma
}

\author{
Ranjana Advani, MD, Weiyun Z. Ai, MD, PhD, and Sandra J. Horning, MD, Stanford, California
}

\section{Key Words}

Prognostic factors, risk-adapted therapy, Hodgkin lymphoma

\begin{abstract}
Although advanced Hodgkin lymphoma is highly curable, balancing the high cure rate with long-term toxicity is challenging. ABVD (doxorubicin [Adriamycin], bleomycin, vinblastine, dacarbazine) is the standard chemotherapy regimen, producing a high cure rate with acceptable toxicity. Stanford V and BEACOPP (bleomycin, etoposide, doxorubicin, cyclophosphamide, vincristine, procarbazine, and prednisone) are new regimens with encouraging results and are undergoing randomized clinical trials. The International Prognostic Score provides a clinical tool that may help identify patients with high-risk disease who may require a more aggressive regimen. Consolidative radiation's role in managing advanced Hodgkin lymphoma is still controversial, but it is most accepted for bulky or residual disease or after brief chemotherapy. The development and integration of newer imaging tools, such as fluorodeoxyglucosepositron emission tomography imaging, may allow a more precise evaluation of disease and help define which patients might benefit from consolidative treatment. (JNCCN 2006;4:241-247)
\end{abstract}

In the United States, advanced Hodgkin lymphoma is defined as stage III or IV disease. In other countries, bulky stage II disease or stage II disease with risk factors (large mediastinal mass $>0.33$ of chest diameter, extranodal sites of disease, elevated erythrocyte sedimentation rate $>50 \mathrm{~mm} / \mathrm{h}$, and $\geq 3$ involved regions) is often managed as advanced disease. Significant progress in the treatment outcome of Hodgkin lymphoma has been made in the past 2 decades. The high cure rate, however, is hampered

From Stanford University Medical Center, Stanford, California. Submitted October 18, 2005; accepted for publication December 7 , 2005.

The authors have no financial interest, arrangement, or affiliation with the manufacturers of any products discussed in the article or their competitors.

Correspondence: Ranjana Advani, MD, Stanford Cancer Center, 875

Blake Wilbur Drive, Stanford, CA 94305 . E-mail: radvani@stanford.edu by significant therapy-related long-term side effects. The goal of current treatment strategies is to cure the disease with minimal toxicity. A risk-adapted approach can help identify patients with high-risk disease or poor prognostic features, and determine appropriate treatment. This article discusses the current management approach to advanced Hodgkin lymphoma.

\section{Chemotherapy for Advanced Hodgkin Lymphoma}

\section{Historical Perspective}

Advanced Hodgkin lymphoma was a uniformly fatal disease until 1964, when the MOPP (mechlorethamine, vincristine, procarbazine, and prednisone) multi-agent regimen was introduced. MOPP produced a response rate of $80 \%$, with $54 \%$ of the patients continuously free of disease at a median follow-up of 14 years. ${ }^{1,2}$ However, successful treatment with MOPP was associated with significant long-term side effects, specifically sterility and secondary leukemia. ${ }^{1,2}$

Variations of MOPP, such as MVPP (mechlorethamine, vinblastine, procarbazine, and prednisone), BCVPP (carmustine, cyclophosphamide, vinblastine, procarbazine, and prednisone), and ChlVPP (chlorambucil, vinblastine, procarbazine, and prednisone), have been developed and tested in clinical trials. These variations did not yield superior efficacy to MOPP or reduce the risk for long-term toxicity, particularly secondary leukemia and myelodysplastic syndromes (MDS). ${ }^{3-5}$

In 1973, Bonadonna et al. ${ }^{6}$ at the National Cancer Institute of Milan developed the ABVD (doxorubicin [Adriamycin], bleomycin, vinblastine, dacarbazine) regimen.This regimen and its variations were then tested against MOPP in large clinical trials in Europe and North America. The landmark Cancer and Leukemia Group B 
Advani et al.

study showed that therapy with ABVD alone and MOPP alternating with ABVD had similar efficacy in both complete response (CR) rate ( $82 \%$ vs. $83 \%)$ and failure-free survival (FFS) (61\% vs. 65\%). Both regimens were superior to therapy with MOPP alone, which had a CR rate of $67 \%$ and FFS of $50 \%(P=$ .002).7 Overall survival (OS) rates were similar for MOPP, ABVD, or alternating MOPP/ABVD, perhaps because of effective salvage therapy. Although ABVD and alternating MOPP/ABVD are equally effective, ABVD had less acute toxicity than the alternating regimen. Mature data at a median follow-up of 14 years confirmed the superiority of ABVD-alone and alternating $\mathrm{MOPP} / \mathrm{ABVD}$ regimens to MOPP, showing a $45 \%$ to $50 \%$ progression-free survival (PFS) and $65 \%$ OS. ${ }^{8}$

MOPP-ABVD hybrid regimens were also developed and tested. ${ }^{9}$ These regimens had equal efficacy to the MOPP/ABVD alternating regimen and were superior in CR rates ( $83 \%$ vs. $75 \% ; P=.02)$ and $F F S$ (64\% vs. $54 \% ; P=.01)$ to a sequential regimen of MOPP followed by ABVD. ${ }^{10}$ The 5-year FFS after ABVD-alone and MOPP-ABV-hybrid regimens were also similar in the Intergroup trial $(63 \%$ vs. $66 \% ; P=$ .42 ), but more hematologic toxicity and treatmentrelated deaths occurred in the hybrid arm. Another concern was the incidence of second malignancies, particularly MDS and secondary leukemia. The increased toxicity in this trial led to its early closure. ${ }^{11}$

Based on the mature results of these randomized clinical trials and a favorable toxicity profile, ABVD is considered the standard treatment for advanced Hodgkin lymphoma. The incidence of sterility and leukemia are much less with ABVD compared with MOPP, though symptomatic pulmonary fibrosis secondary to ABVD has been reported in $6 \%$ of patients. ${ }^{12}$

\section{International Prognostic Score for Advanced Hodgkin Lymphoma}

The International Prognostic Scoring system (IPS) was developed by the International Prognostic Factors Project on Advanced Hodgkin's Disease. ${ }^{13}$ This clinically relevant prognostic scoring system is based on data from 5,023 patients treated with combination chemotherapy with or without radiation. The study identified 7 independent factors ( 3 clinical and 4 laboratory) that adversely impacted the likelihood of freedom from progression (FFP) and OS: 1) a serum albumin less than $4 \mathrm{~g} / \mathrm{dL} ; 2$ ) hemoglobin less than 10.5 $\mathrm{g} / \mathrm{dL} ; 3$ ) male gender; 4) stage IV disease; 5) age of 45 years or order; 6) white cell count of $15,000 / \mathrm{mm}^{3}$ or more; and 7) lymphocyte count less than $600 / \mathrm{mm}^{3}$ or $8 \%$ of the total white cell count. The distribution of patients according to the IPS was $30 \%, 50 \%$, and $20 \%$ for risk factors 0 to $1,2-3$, and 4-7, respectively. Patients with no risk factors had a 5-year FFP rate of $80 \%$. Each additional risk factor decreased the likelihood of FFP at 5 years by $7 \%$. The highest-risk group (5 or more risk factors), which represented fewer than $20 \%$ of patients, had only a $42 \%$ FFP, suggesting that advanced-stage Hodgkin lymphoma may be heterogeneous. The IPS is therefore not only a basis for a riskadapted treatment approach, but also a useful clinical tool that allows more precise comparison of therapies.

\section{New Chemotherapy Regimens for Advanced Hodgkin Lymphoma}

ABVD is the standard regimen for advanced Hodgkin lymphoma, with a CR rate of $80 \%$ to $90 \%$. However, at 5 years, $40 \%$ of patients relapse and $15 \%$ to $25 \%$ die of the disease or treatment complications. ${ }^{8}$ Two major new regimens have emerged to further improve outcomes for patients with advanced Hodgkin lymphoma: Stanford V (mechlorethamine, doxorubicin, vinblastine, vincristine, bleomycin, etoposide, prednisone) developed by the Stanford group, and BEACOPP (bleomycin, etoposide, doxorubicin, cyclophosphamide, vincristine, procarbazine, prednisone) developed by the German Hodgkin Lymphoma Study Group (GHLSG).

The goal of the Stanford V regimen is to maintain or improve the cure rate while minimizing the acute and long-term toxicities. The regimen consists of a 12-week course of chemotherapy given in combination with consolidative irradiation ( $36 \mathrm{~Gy})$ to sites of bulky disease $(\geq 5 \mathrm{~cm})$ and macroscopic splenic involvement. Radiotherapy starts 2 weeks after chemotherapy is completed. Compared with ABVD or hybrid regimens, Stanford V maintains or increases dose-intensity of individual drugs but reduces the cumulative doses of bleomycin, doxorubicin, and nitrogen mustard, and omits procarbazine. This regimen may reduce or prevent pulmonary and cardiac dysfunction, sterility, and the incidence of secondary leukemia. To reduce the risks for breast cancer and long-term cardiac or vascular toxicity, researchers 
Management of Advanced Stage Hodgkin Lymphoma

decreased the radiotherapy fields and omitted the axillae and high neck from treatment unless they were sites of bulky disease. Chemotherapy intensity was supported by growth factor use after the first dosereduction or treatment delay.

Mature results from a phase II study of 162 patients at Stanford report an FFP of $89 \%$ and OS of $96 \%$, with a median follow-up of 5.4 years. Patients with up to 2 risk factors according to the IPS had a 94\% FFP, compared with $75 \%$ for those with 3 or more risk factors. ${ }^{14}$ The OS for this high-risk group was more than $90 \%$ because of effective salvage. Neutropenia and constipation were the main toxicities of this regimen; no bleomycin toxicity or radiation pneumonitis was observed. In addition, no secondary MDS/leukemia or lymphoma has yet been reported. Unlike the older MOPP regimen or the more recent BEACOPP combination, Stanford V preserves fertility. A recent update reported 72 posttreatment conceptions (excluding those involving pretreatment semen or embryo cryopreservation), with 65 live births. ${ }^{15}$

A phase II study from Memorial Sloan-Kettering Cancer Center (MSKCC), a pilot study by the Eastern Cooperative Oncology Group (ECOG), and a multicenter study from Italy have reported similar outcomes. ${ }^{16-18}$ In both the Stanford and MKSCC series, approximately $50 \%$ to $60 \%$ of patients for whom treatment with the Stanford V regimen failed underwent successful salvage with secondary treatment. Two ongoing randomized trials are comparing the Stanford $\mathrm{V}$ and ABVD treatments in patients with advanced disease. The ECOG 2496 Intergroup trial is comparing the Stanford V program (chemotherapy plus radiation) with ABVD plus radiation for bulky mediastinal disease, and a United Kingdom study is comparing the Stanford V chemotherapy regimen plus radiation with ABVD plus radiation. The latter trial followed a pilot study comparing Stanford V plus radiation with ABVD plus radiation (to sites $>5 \mathrm{~cm}$ or macroscopic splenic disease) that showed excellent efficacy results for both regimens. ${ }^{19}$ Mature results from these ongoing trials will help evaluate the long-term effects for cure and complications.

Recently, another group from Italy (Intergruppo Italiano Linfomi) compared ABVD with a modified Stanford V program or a multi-agent chemotherapy regimen consisting of mechlorethamine, vincristine, procarbazine, prednisone, epidoxorubicin, bleomycin, vinblastine, lomustine, doxorubicin, and vindesine (MOPPEBVCAD).$^{20}$ In this study, the 5-year FFS and PFS rates for the modified Stanford V arm were inferior to those for the other 2 regimens, with no differences in OS. These results are difficult to interpret because the response evaluation that determined if patients would continue on the study arm was performed at different times: after 8 weeks of Stanford V chemotherapy, 16 weeks for ABVD, and 24 weeks for MOPPEBVCAD. Also, the modifications to radiotherapy were substantial, including a limit on the number of treated sites, a different definition of bulk, and a delay in initiating radiotherapy to a median of 6 weeks. Thus, this treatment approach differed significantly from the Stanford V regimen, underscoring the need to enroll patients in the ongoing phase III trials described earlier.

To improve the outcome of advanced Hodgkin disease, the GHLSG used a dose-escalation approach based on a mathematical model. ${ }^{21}$ In the 3-arm HD9 trial, 1,201 patients with newly diagnosed Hodgkin disease in unfavorable stage IIB, IIIA, IIIB, or IV were randomized to receive 8 cycles of alternating COPP and ABVD, 8 cycles of baseline BEACOPP, or $8 \mathrm{cy}$ cles of escalated BEACOPP.2 At the end of treatment, approximately two thirds of these patients received 36 Gy radiotherapy to initial bulky $(>5 \mathrm{~cm})$ or residual disease. This intense regimen was administered with growth factor support. At a median follow-up of 5 years, the 2 BEACOPP regimens were superior to the COPP/ABVD regimen in CR, FFP, and OS. The escalated BEACOPP was superior to baseline BEACOPP in FFP ( $87 \%$ vs. $76 \% ; P<.001)$, but not OS (91\% vs. $86 \% ; P=.06)$.

Although the 2 BEACOPP regimens were superior to COPP/ABVD in all IPS-based prognostic groups, the poor-risk group (patients with 4 to 7 risk factors) showed the most pronounced difference in OS. Dose-intense therapy only showed benefit for patients younger than 60 years, and was associated with increased but manageable hematologic toxicity. Three percent of patients died of acute treatment-related complications. Experts anticipate this regimen will cause sterility in young adults. ${ }^{23}$ Four secondary leukemias were reported in the standard BEACOPP arm and 9 in the escalated BEACOPP arm, versus 1 in the COPP/ABVD arm..$^{22}$ Efforts are ongoing in the GHLSG to reduce these toxicities, specifically secondary leukemia. 
Advani et al.

Based on the HD9 trial's treatment results with escalated BEACOPP and the assumption that many patients were overtreated, the goal of the HD12 trial was to reduce acute and long-term toxicity by administering 4 cycles of escalated BEACOPP followed by 4 cycles of baseline BEACOPP. Patients were randomized to undergo observation or radiotherapy to sites with initial bulky disease $(\geq 5 \mathrm{~cm})$ or residual disease after chemotherapy. Preliminary results from this trial show 4 cycles of standard BEACOPP plus 4 cycles of escalated BEACOPP have similar efficacy compared with 8 cycles of escalated BEACOPP. ${ }^{24}$ Another variation, the BEACOPP -14 regimen (baseline BEACOPP administered every 14 days for 8 cycles with growth factor support), has also been used to decrease toxicity compared with escalated BEACOPP, while maintaining efficacy. ${ }^{25}$ Results for this pilot study are comparable with the results of the HD9 trial, and no acute leukemia or myelodysplasia was seen at a short median follow-up of 34 months. An ongoing study (the HD15 trial) is currently evaluating this regimen, comparing 6 cycles of escalated BEACOPP, 8 cycles of escalated BEACOPP, and 8 courses of BEACOPP. 14 , with radiotherapy given only to patients with positron emission tomography (PET)-positive residual tumors.

An ongoing European Organization for Research and Treatment of Cancer (EORTC) trial is comparing 8 cycles of ABVD with 4 cycles of standard BEACOPP plus 4 cycles of escalated BEACOPP in patients with an IPS of 3 or more. Longer follow-up is needed to determine if the increased toxicity of escalated BEACOPP regimens is justified. With mature followup, other late complications may be recognized, such as the $13 \%$ incidence of avascular necrosis of the hip recently reported in a study from the Czech Republic. ${ }^{26}$

The roles of high-dose therapy and autologous bone marrow transplantation have also been evaluated in patients with high-risk disease. Although the definition of high-risk disease varies, 2 trials randomized patients who had experienced complete or partial remission after primary chemotherapy for advanced Hodgkin lymphoma with adverse prognostic factors to receive continued standard- or high-dose chemotherapy and hematopoietic stem cell transplantation. The studies showed no difference in outcome between the groups. ${ }^{27,28}$ Therefore, high-dose chemotherapy with stem cell support is reserved for patients with primary refractory or relapsed Hodgkin lymphoma.

\section{Role of Radiation in Advanced Hodgkin Lymphoma}

Using radiotherapy as an adjuvant to chemotherapy in patients with stage III/IV disease is justified by observations of relapse in previously involved unirradiated sites, often bulky nodes, even in patients with stage IV disease. ${ }^{29}$ Because radiation can provide local control, many studies have incorporated it into combined-modality therapies. However, its role is controversial because most earlier reports used older chemotherapy regimens, such as MOPP, and relatively high-dose large radiotherapy fields, such as subtotal lymphoid irradiation and total lymphoid irradiation. ${ }^{30,31}$ These factors most likely contributed to the excess morbidity and mortality seen in Hodgkin lymphoma survivors, mainly therapy-related second cancers and cardiac disease. ${ }^{32}$

The EORTC 20884 trial randomized patients who experienced a CR after treatment with 6 to 8 cycles of MOPP-ABVD chemotherapy to undergo 25 Gy involved-field radiotherapy (IFRT) versus no further therapy. No differences in freedom from treatment failure (FFTF) or OS were identified. However, patients in this trial who experienced a partial remission underwent treatment with 30 Gy IFRT. Notably, the FFTF and OS rates for this group were similar to those of patients who had experienced a CR, suggesting that consolidative radiation may be beneficial in patients who did not experience a CR with chemotherapy. ${ }^{33}$

The role of radiotherapy when ABVD is used as chemotherapy has been tested in a randomized study in which the FFP and OS favor the combinedmodality arm..$^{34}$ This study is difficult to extrapolate to adults because half of the patients were younger than 15 years and had early-stage disease. Most also had a mixed cellularity subtype.

The role of radiation therapy became even more controversial in advanced-stage patients after the advent of more intense chemotherapy regimens. The GHLSG HD12 trial randomized adult patients to undergo either consolidative radiotherapy (to sites of initial bulk $>5 \mathrm{~cm}$ or residual disease) or observation after BEACOPP ( 8 escalated or 4 escalated plus 4 standard). An interim analysis reported no advantage for radiotherapy. ${ }^{24}$ However, $13 \%$ of patients who were not assigned to undergo radiation did because of concern for residual disease, leaving the benefits of adjuvant irradiation in this setting unknown. 
Management of Advanced Stage Hodgkin Lymphoma

Thus, the advantage of radiation seems minimal for adults experiencing CR after a full course of chemotherapy (6-8 cycles). Radiotherapy offers a modest advantage for patients experiencing partial remission and those with bulky disease. The GHLSG HD15 trial is currently investigating the advantages of radiation tailored to sites of disease that remain fluorodeoxyglucose-positron emission tomography (FDGPET)-positive after intense chemotherapy (escalated BEACOPP 6 vs. 8 cycles, or BEACOPP-14).

When a brief chemotherapy approach is used, such as the Stanford V regimen, timely radiation therapy is an integral part of therapy. This was evident in the inferior performance of Stanford V without standardized radiotherapy in an Italian 3-arm study. ${ }^{20}$

\section{Role of PET}

As the management of Hodgkin lymphoma becomes more targeted, treatment intensity must be tailored to produce excellent cure rates while limiting longterm toxicities. FDG-PET imaging has recently been used in the management of Hodgkin lymphoma. Several studies have evaluated the role of FDG-PET for initial staging as compared to other imaging techniques. Most studies are retrospective, include patients with Hodgkin lymphoma and non-Hodgkin's lymphoma, and are limited by the lack of histologic confirmation. PET has a higher sensitivity and resolution compared with gallium scans. ${ }^{35,36}$ Compared with computed tomography (CT) scanning, PET is more sensitive in detecting nodal and extranodal disease (especially bone and spleen), although CT-positive PET-negative lesions occur in a few cases. High FDG uptake in brown fat or muscle can be seen in the head and neck area, although physiologic uptake in the renal and gastrointestinal tract can obscure evaluation at these sites. Incorporating PET in the initial staging can change the stage in $10 \%$ to $20 \%$ of patients. In a study evaluating 88 patients with Hodgkin lymphoma, $7 \%$ of patients had a false-negative PET that could have led to potentially inappropriate therapy. Therefore, PET should not be used as a sole modality for staging, but in conjunction with a CT. ${ }^{37}$

When therapy is complete, PET is useful in distinguishing nonmalignant tissue from residual tumor in patients who present with bulky disease and show residual masses on standard imaging techniques. Several studies have reported a high negative predic- tive value (NPV) $(81 \%-100 \%){ }^{38-40}$ Relapses are infrequent and rarely occur within the first year of completing therapy, suggesting a low tumor burden undetectable by PET. However, the risk for late relapse cannot be excluded, and these patients may benefit from repeated follow-up scans. Alternative therapy should be considered when abnormal FDG uptake persists at sites of initial involvement after first-line therapy is completed and coexisting inflammation is not highly suspected. In contrast with the NPV, the positive predictive value of PET varies from $25 \%$ to $100 \%$, perhaps because of nonmalignant conditions such as thymic hyperplasia, granulomatous disease, or infection. . $^{38-40}$

Physiologic thymic uptake, which must be distinguished from residual disease, has been reported in up to $30 \%$ of patients. ${ }^{39}$ The timing of PET scanning is also important because inflammation from radiotherapy may provide false-positive results. Recently, combined CT and PET have improved the accuracy correlation between anatomic sites and increased signal. The GHLSG HD15 trial, which selects patients for consolidative radiation based on residual PETpositive disease, is testing the usefulness of PET in providing rational radiation use.

\section{Summary}

With current therapy, $80 \%$ of patients with advanced Hodgkin lymphoma will be cured, but balancing efficacy with complications by adopting a risk-adapted strategy remains challenging. ABVD is still the gold standard against which newer therapies are evaluated. Two intense regimens have recently been developed with encouraging results. In a randomized trial, the GHLSG reported superior efficacy of the escalated BEACOPP regimen, although this must be weighed against substantial treatment-related toxicities. Data from the Stanford V regimen are also encouraging, specifically the lack of secondary MDS and preservation of fertility. Ongoing randomized trials in the United States and United Kingdom are comparing this regimen with ABVD.

A risk-adapted approach may be useful in managing these patients. For low-risk patients (approximately $30 \%$ ), less-toxic therapy such as ABVD is highly curative. For most intermediate-risk patients, ABVD and Stanford V are both reasonable choices because they preserve fertility and have low risk for development of 
Advani et al.

secondary leukemia. The likelihood of cure with primary therapy is less than $50 \%$ for the $20 \%$ of patients with poor-risk disease ( $\geq 4$ risk factors), and more intensified treatments may be required. Some physicians and patients may prefer to use a less-intense regimen because second-line treatment is potentially curative if relapse occurs, thus sparing patients from the initial intense regimen. Mature data from ongoing trials will help define the role of these newer therapies. OS and quality of life are still important end points. Continued identification of biologic features and correlation with clinical prognostic factors will help characterize disease subsets to which therapy can be further tailored.

\section{References}

1. Longo $\mathrm{D}$, Young R, Wesley $\mathrm{M}$, et al. Twenty years of MOPP therapy for Hodgkin's disease. J Clin Oncol 1986;4:1295-1306.

2. DeVita VT Jr, Simon RM, Hubbard SM, et al. Curability of advanced Hodgkin's disease with chemotherapy. Long-term follow-up of MOPP-treated patients at the National Cancer Institute. Ann Intern Med 1980;92:587-595.

3. Vose J, Bierman P, Anderson J, et al. CHLVPP chemotherapy with involved-field irradiation for Hodgkin's disease: favorable results with acceptable toxicity. J Clin Oncol 1991;9:1421-1425.

4. Harding MJ, McNulty LJ, Paul J, et al. Mechlorethamine, vinblastine, procarbazine and prednisolone (MVPP) for advanced Hodgkin's disease. Eur J Cancer 1991;27:1002-1006.

5. Hancock BW, Vaughan Hudson G, Vaughan Hudson B, et al. British National Lymphoma Investigation randomised study of MOPP (mustine, Oncovin, procarbazine, prednisolone) against LOPP (Leukeran substituted for mustine) in advanced Hodgkin's disease-long term results. Br J Cancer 1991;63:579-582.

6. Bonadonna G, Zucali R, Monfardini S, et al. Combination chemotherapy of Hodgkin's disease with adriamycin, bleomycin, vinblastine, and imidazole carboxamide versus MOPP. Cancer 1975;36:252-259.

7. Canellos GP, Anderson JR, Propert KJ, et al. Chemotherapy of advanced Hodgkin's disease with MOPP, ABVD, or MOPP alternating with ABVD. N Engl J Med 1992;327:1478-1484.

8. Canellos GP, Niedzwiecki D. Long-term follow-up of Hodgkin's disease trial. N Engl J Med 2002;346:1417-1418.

9. Viviani S, Bonadonna $G$, Santoro A, et al. Alternating versus hybrid MOPP and ABVD combinations in advanced Hodgkin's disease: ten-year results. J Clin Oncol 1996;14:1421-1430.

10. Glick JH, Young ML, Harrington D, et al. MOPP/ABV hybrid chemotherapy for advanced Hodgkin's disease significantly improves failure-free and overall survival: the 8-year results of the intergroup trial. J Clin Oncol 1998;16:19-26.

11. Duggan DB, Petroni GR, Johnson JL, et al. Randomized comparison of $A B V D$ and MOPP/ABV hybrid for the treatment of advanced Hodgkin's disease: report of an intergroup trial. J Clin Oncol 2003;21:607-614.
12. Viviani S, Santoro A, Ragni G, et al. Gonadal toxicity after combination chemotherapy for Hodgkin's disease. Comparative results of MOPP vs ABVD. Eur J Cancer Clin Oncol 1985;21:601-605.

13. Hasenclever D, Diehl V. A prognostic score for advanced Hodgkin's disease. International Prognostic Factors Project on Advanced Hodgkin's Disease. N Engl J Med 1998;339:1506-1514.

14. Horning SJ, Hoppe RT, Breslin S, et al. Stanford V and radiotherapy for locally extensive and advanced Hodgkin's disease: mature results of a prospective clinical trial. J Clin Oncol 2002;20:630-637.

15. Horning SJ, Hoppe R, Advani R, et al. Efficacy and late effects of Stanford V chemotherapy and radiotherapy in untreated Hodgkin's disease: mature data in early and advanced stage patients [abstract]. Blood 2004;104 Abstract 308.

16. Yahalom J, Edwards-Bennet $S$, Jacobs J, et al. Stanford V and radiotherapy for advanced and locally extensive Hodgkin's disease (HD): the Memorial Sloan-Kettering Cancer Center (MSKCC) experience [abstract]. Blood 2003 Abstract 1459.

17. Horning SJ, Williams J, Bartlett NL, et al. Assessment of the Stanford $\mathrm{V}$ regimen and consolidative radiotherapy for bulky and advanced Hodgkin's disease: Eastern Cooperative Oncology Group Pilot Study E1492. J Clin Oncol 2000;18:972-980.

18. Aversa SM, Salvagno L, Soraru M, et al. Stanford V regimen plus consolidative radiotherapy is an effective therapeutic program for bulky or advanced-stage Hodgkin's disease. Acta Haematol 2004;112:141-147.

19. Johnson $P$, Hoskin $P$, Horwich $A$, et al. Stanford V (SV) regimen versus ABVD for the treatment of advanced Hodgkin lymphoma (HL): results of a UK NCRI/LTO randomised phase II trial [abstract]. Blood 2004;104 Abstract 311.

20. Gobbi PG, Levis A, Chisesi T, et al. ABVD versus modified Stanford $\mathrm{V}$ versus MOPPEBVCAD with optional and limited radiotherapy in intermediate- and advanced-stage Hodgkin's lymphoma: final results of a multicenter randomized trial by the Intergruppo Italiano Linfomi. J Clin Oncol 2005;23:9198-9207.

21. Hasenclever D, Loeffler M, Diehl V. Rationale for dose escalation of first line conventional chemotherapy in advanced Hodgkin's disease. German Hodgkin's Lymphoma Study Group. Ann Oncol 1996; 7[suppl 4]:95-98.

22. Diehl V, Franklin J, Pfreundschuh M, et al. Standard and increaseddose BEACOPP chemotherapy compared with COPP-ABVD for advanced Hodgkin's disease. N Engl J Med 2003;348:2386-2395.

23. Connors JM: State-of-the-art therapeutics: Hodgkin's lymphoma. J Clin Oncol 2005;23:6400-6408.

24. Diehl V, Brillant C, Franklin J, et al. BEACOPP chemotherapy for advanced Hodgkin's disease: results of further analyses of the HD9. and HD12-trials of the German Hodgkin Study Group (GHSG) [abstract]. Blood 2004;104 Abstract 307.

25. Sieber M, Bredenfeld $H$, Josting A, et al. 14-day variant of the bleomycin, etoposide, doxorubicin, cyclophosphamide, vincristine, procarbazine, and prednisone regimen in advanced-stage Hodgkin's lymphoma: results of a pilot study of the German Hodgkin's Lymphoma Study Group. J Clin Oncol 2003;21:1734-1739.

26. Markova J, Zidka M, Kozak T. Osteonecrosis as a complication of treating Hodgkin's lymphoma after BEACOPP chemotherapy. Ann Oncol 2005;16(Suppl 5). Abstract 269.

27. Federico M, Bellei M, Brice P, et al. High-dose therapy and autologous stem-cell transplantation versus conventional therapy for patients with advanced Hodgkin's lymphoma responding to frontline therapy. J Clin Oncol 2003;21:2320-2325.

28. Proctor SJ, Mackie M, Dawson A, et al. A population-based study of intensive multi-agent chemotherapy with or without autotrans- 
Management of Advanced Stage Hodgkin Lymphoma

plant for the highest risk Hodgkin's disease patients identified by the Scotland and Newcastle Lymphoma Group (SNLG) prognostic index. A Scotland and Newcastle Lymphoma Group study (SNLG HD III). Eur J Cancer 2002;38:795-806.

29. Young RC, Canellos GP, Chabner BA, et al. Patterns of relapse in advanced Hodgkin's disease treated with combination chemotherapy. Cancer 1978;42:1001-1007.

30. Loeffler M, Brosteanu O, Hasenclever D, et al. Meta-analysis of chemotherapy versus combined modality treatment trials in Hodgkin's disease. International Database on Hodgkin's Disease Overview Study Group. J Clin Oncol 1998;16:818-829.

31. Ferme C, Sebban C, Hennequin C, et al. Comparison of chemotherapy to radiotherapy as consolidation of complete or good partial response after six cycles of chemotherapy for patients with advanced Hodgkin's disease: results of the groupe d'etudes des lymphomes de l'Adulte H89 trial. Blood 2000;95:2246-2252.

32. Hancock SL, Hoppe RT, Horning SJ, et al. Intercurrent death after Hodgkin disease therapy in radiotherapy and adjuvant MOPP trials. Ann Intern Med 1988;109:183-189.

33. Aleman BM, Raemaekers JM, Tirelli U, et al. Involved-field radiotherapy for advanced Hodgkin's lymphoma. N Engl J Med 2003; 348:2396-2406.

34. Laskar S, Gupta T, Vimal S, et al. Consolidation radiation after complete remission in Hodgkin's disease following six cycles of doxoru- bicin, bleomycin, vinblastine, and dacarbazine chemotherapy: is there a need? J Clin Oncol 2004;22:62-68.

35. Friedberg JW, Fischman A, Neuberg D, et al. FDG-PET is superior to gallium scintigraphy in staging and more sensitive in the followup of patients with de novo Hodgkin lymphoma: a blinded comparison. Leuk Lymphoma 2004;45:85-92.

36. Wirth A, Seymour JF, Hicks RJ, et al. Fluorine-18 fluorodeoxyglucose positron emission tomography, gallium-67 scintigraphy, and conventional staging for Hodgkin's disease and non-Hodgkin's lymphoma. Am J Med 2002;112:262-268.

37. Naumann R, Beuthien-Baumann B, Reiss A, et al. Substantial impact of FDG PET imaging on the therapy decision in patients with early-stage Hodgkin's lymphoma. Br J Cancer 2004;90:620-625.

38. Spaepen K, Stroobants S, Dupont P, et al. Can positron emission tomography with [(18)F]-fluorodeoxyglucose after first-line treatment distinguish Hodgkin's disease patients who need additional therapy from others in whom additional therapy would mean avoidable toxicity? Br J Haematol 2001;115:272-278.

39. Jerusalem G, Beguin Y, Fassotte MF, et al. Early detection of relapse by whole-body positron emission tomography in the follow-up of patients with Hodgkin's disease. Ann Oncol 2003;14:123-130.

40. Weihrauch MR, Re D, Scheidhauer K, et al. Thoracic positron emission tomography using $18 \mathrm{~F}$-fluorodeoxyglucose for the evaluation of residual mediastinal Hodgkin disease. Blood 2001;98:2930-2934. 